\title{
Anatomia e Histologia das Glândulas Salivares nos Triatomíneos
}

\section{Dyrce Lacombe}

\begin{abstract}
Departamento de Entomologia, Instituto Oswaldo Cruz, Estrada da Covanca nº 56, 22735-200 Jacarepaguá, Rio
\end{abstract} de Janeiro, RJ, Brasil

\begin{abstract}
Anatomy and Histology of Salivary Glands of Triatomine Bugs - Histological studies upon the salivary glands of ten species of triatomine bugs were performed looking for their number and structural organization in different genera. It was possible to evaluate the celular epithelium type of each gland, as well as the merocrine and apocrine secretions of the glands. Secretion run until the hilo and after to salivary pump and hypofaringe. The glandular components, D1, D2 and D3 are always present in the Triatoma, Panstrongylus and Diptelogaster but in Rhodnius there are only the first two pairs of glands. The salivary channels and the hilo are analyzed by histology. The whole pair D3 has a clear valve that regularizes the exit of the secretions to the hilo. According to the genus the valves appear in different locations. They have low and dense epithelium, and their nucleus are rich in chromatin. The secondary channels leaving these valves, are very different, with clear chitinous ringer, low level of chromatin in the nucleus and homogeneous cytoplasm.
\end{abstract}

Key words: salivary glands - Triatominae - histology

As glândulas salivares têm grande diversificação quanto ao número, tamanho, forma e situação nos diferentes triatomíneos. Em geral estão situadas no tórax, onde encontram espaço suficiente para seu desenvolvimento. O deslocamento para a região abdominal resulta dos eventuais movimentos peristálticos dos órgãos vizinhos. Os estudos sobre a anatomia, microanatomia e histologia do sistema glandular de hemípteros foi, por muitos anos, alvo de atenção de vários pesquisadores (Bordas 1905, Bugnion e Popoff 1908, Fauré-Freemier 1910, Barreto 1920, Puri 1924, Weber 1930, Wigglesworth 1939, Baptist 1941, Barth 1954). Mais recentemente, alguns pesquisadores vêm se dedicando ao estudo das glândulas salivares desde o ponto de vista anatômico até o ultraestrutural, através de microscopia eletrônica de transmissão e de varredura, além de oferecerem detalhes sobre a constituição química da saliva, particularmente de insetos hematófagos (Ribeiro 1987, Konig et al. 1993, Garcia et al. 1994, Stark \& James 1996, Pereira et al. 1996, Santos et al. 1997, Ribeiro et al. 1998, Sun et al. 1998). O presente estudo, porém, está limitado às observações anatômicas e histológicas das glândulas salivares de quatro gêneros e dez espécies de triatomíneos em conjunto, assunto muito pouco estudados nas últimas décadas. Os trabalhos de Baptist (1941) e Barth (1954)

Recebido em 26 de Agosto de 1998 Aceito em 22 de Abril de 1999 nos serviram de orientação durante os estudos. A nomenclatura usada para as glândulas salivares D1, D2 e D3 - segue aquela mencionada por Barth (1954) durante seus estudos nas glândulas salivares de Triatoma infestans. O par D1 corresponde às glândulas principais, o D2 às glândulas suplementares e o par D3 apenas às glândulas acessórias. Para alguns autores (ex. Santos et al. 1997), as glândulas D1 e D2 corresponderiam aos lobos anterior e posterior da glândula principal dos triatomíneos. Barth (1954) demonstra a importância dos três pares de glândulas na formação da saliva desses insetos. Com base em cortes histológicos seriados e nas observações feitas pela autora (Lacombe 1962) sobre o sistema traqueal nos triatomíneos, conclui-se que as glândulas D3 são muito ativas, recebendo intensa oxigenação, não devendo ser consideradas apenas como simples reservatórios de saliva. A presença de uma verdadeira válvula na glândula D3, fato aqui estudado, reforça a conclusão sobre a importância desta glândula na formação da saliva, em triatomíneos.

\section{MATERIAIS E MÉTODOS}

Para este estudo utilizamos cerca de 300 exemplares de triatomíneos, adultos e de ambos os sexos. As espécies estudadas foram: T. vitticeps, $T$. pessoai, T. infestans, T. pseudomaculata, T. brasiliensis, T. pallidpenis, Panstrongylus megistus, Diptelogaster maxima, Rhodnius prolixus e $R$. neglectus. Cerca de 1,5\% dos exemplares nos foram fornecidos pelo Laboratório $\mathrm{Na}$ cional e Internacional de Referência em Taxonomia 
de Triatomíneos, do Departamento de Entomologia do Instituto Oswaldo Cruz (IOC). Os demais insetos foram provenientes da criação organizada pela Dra. Alina Szumewicz, do Departamento de Medicina Tropical, IOC. Todos os exemplares de cada espécie foram separados em dois lotes. O primeiro lote foi destinado aos estudos de anatomia e microana-tomia e o segundo reservado para as análises histológicas.

De início, fixamos o exemplar, narcotizado com éter, em uma placa de Petri forrada com camada de parafina, embebendo-o em uma solução para dissecção $(0,7 \% \mathrm{KCl}+0,3 \% \mathrm{NaCl})$. Cortamos os bordos laterais dos esternitos, aprofundando até à região cervical. A seguir, retiramos os tergitos expondo as glândulas salivares, parte do aparelho digestivo, o vaso dorsal, o corpo gorduroso, o sistema traqueal e a musculatura.

As glândulas salivares são facilmente destacadas na região mediana dorsal do tórax. No lote 1 , destinado à anatomia e microanatomia, as glândulas foram retiradas e colocadas no líquido fixador de Bouin, por 30 min., e depois lavadas em soro fisiológico. Neste momento observamos a forma das glândulas, a relação com outros órgãos, o comprimento, a localização e a coloração. Nos estudos de microanatomia, estas glândulas foram desidratadas e colocadas em xilol a 100\%, durante $6 \mathrm{~h}$. Depois foram transferidas para o salicilato de metila e benzoato de benzila (1:1) onde permaneceram por $24 \mathrm{~h}$. Após a passagem pelo xilol, por $24 \mathrm{~h}$, foram colocadas entre lâminas e lamínulas e montadas em Entelan (Merk).

No lote 2, referente aos estudos histológicos, as glândulas salivares e uma parte do aparelho digestivo foram retiradas e de imediato colocadas nos fixadores Bouin, Susa, Carnoy, Flemming e Zenker. Os procedimentos adotados foram os convencionais, utilizando-se os fixadores e métodos descritos por Romein (1928), Barth (1953), Pearse (1960) e Thompson (1966). Subsequientemente, as glândulas foram incluídas em parafina Histoseck (Merck) e os blocos feitos no aparelho Tissue Teck. Foram feitos cortes histológicos seriados, longitudinais e transversais, com $5 \mu \mathrm{m}, 7 \mu \mathrm{m}$ e $10 \mu \mathrm{m}$ de espessura. As colorações empregadas foram Gallocianina, Hematoxilina férrica de Heidenhein, Cromotrop 2R, Vermelho de Congo + Orange GG, Hematoxilina segundo Delafield, Mallory e Erlich, Eosina e Verde Naftol.

\section{RESULTADOS E DISCUSSÃO}

Em todas as espécies de triatomíneos estudadas sempre encontramos os três pares de glândulas salivares, com exceção do gênero Rhodnius que não apresenta a típica glândula D3. Devemos ressaltar que em $T$. vitticeps e $T$. brasiliensis os três pares de glândula são mais evidentes em relação às demais espécies. Após a retirada dos tergitos e esternitos de $T$. vitticeps, vemos com clareza os três pares de glândulas (Fig. 1: D1, D2 e D3) situadas próximos ao tubo digestivo e estendendo-se até o início do promesêntero.

De forma geral as glândulas D1 e D2 têm cor branco leitosa ou um pouco amarelada, como ocorre em P. megistus. Entretanto, no gênero Rhodnius as glândulas D1 e D2 são alongadas e de cor avermelhada, o que facilita a sua visualização e extração para estudos bioquímicos (Fig. 2). As colorações das glândulas resultam da presença de secreção na sua luz. O par de glândulas D3, em todos os espécimes do gênero Triatoma, Panstrongylus e Diptelogaster, está situado na região abdominal. As glândulas D1 e D2 se mostram cheias de secreção, enquanto a D3 aparenta estar sempre vazia (Fig. 3). Somente uma análise histoquímica da D3 poderia quantificar a possível secreção protéica ali existente.

Um fator a se considerar nos estudos morfológicos das glândulas salivares é o estado de nutrição do inseto. Em animais famintos, as glândulas estão repletas de secreção, ocasionando a distensão da parede celular e conseqüentemente uma modificação morfológica (Fig. 4). O par de glândulas D3 está sempre afastado das demais, mostrando-se transparente, maior em tamanho e deslocado para a região abdominal do inseto. $\mathrm{O}$ aspecto alongado deste par de glândulas pode ser visto na Fig. 5, onde assinalamos a presença de sua válvula e a posição próxima ao promesêntero. Baptist (1914), em seu estudo sobre a morfologia e fisiologia das glândulas salivares nos hemípteros, refere-se a diminutas vesículas no gênero Rhodnius que se abrem diretamente no par de glândulas D1. Mesmo usando técnicas especiais, com o emprego do ácido ósmico, não foi possível em nosso estudo observar estas estrutura em Rhodnius. Acreditamos que observações ao nível de microscopia eletrônica possam verificar se as as glândulas que chamamos D3 não existem, ou não estão evidentes no gênero Rhodnius, embora sejam acentuadas em Triatoma, Panstrongylus e Diptelogaster.

A estrutura histológica das glândulas D1, D2 e D3 é bem diferente entre si. De um modo geral, o par de glândulas D1 é sempre reniforme em todo os hemípteros estudados. Próximo à visível depressão no corpo dessa glândula encontramos o par D2 e o hilo. A Fig. 6 mostra um corte histológico, transversal, pelas glândulas D1, D2 e o hilo. O epitélio da D1 é simples, alto e bem claro como se pode observar após um corte transversal por esta glândula (Fig. 7: EP). A maioria de suas células é binucleada, o que indica um acréscimo de massa nuclear em relação ao citoplasma. Na Fig. 8 observamos a presença de 
grande quantidade de ergastoplasma, a membrana basal e os núcleos. Estes são pobres em cromatina, binucleados e podem ter um ou mais nucléolos (Fig. 9). As glândulas D1 têm secreção merócrina (Fig. 10: SM) e necessitam de rápida regeneração conforme mostra a Fig. 10, em um corte transversal feito na glândula de T. vitticeps. Este fato também ocorre nas glândulas salivares de outros artrópodes, bem como nas glândulas de cimento dos Balanídeos, onde a constante secreção celular ocasiona a formação de inúmeros núcleos polimorfos com dez ou mais nucléolos. Esses achados levaram Boquet-Vedrine (1970) a elaborar um trabalho sobre as formas de núcleos nas glândulas de cimento desses artrópodes.

A função do par de glândulas D1 é produzir uma substância anticoagulante, que é excretada para o lúmem da glândula junto com parte do epitélio que, subseqüentemnte, se refaz. Toda a secreção fica contida nos vacúolos dispersos no citoplasma. Uma característica da D1 é a abundância de traquéolas, o
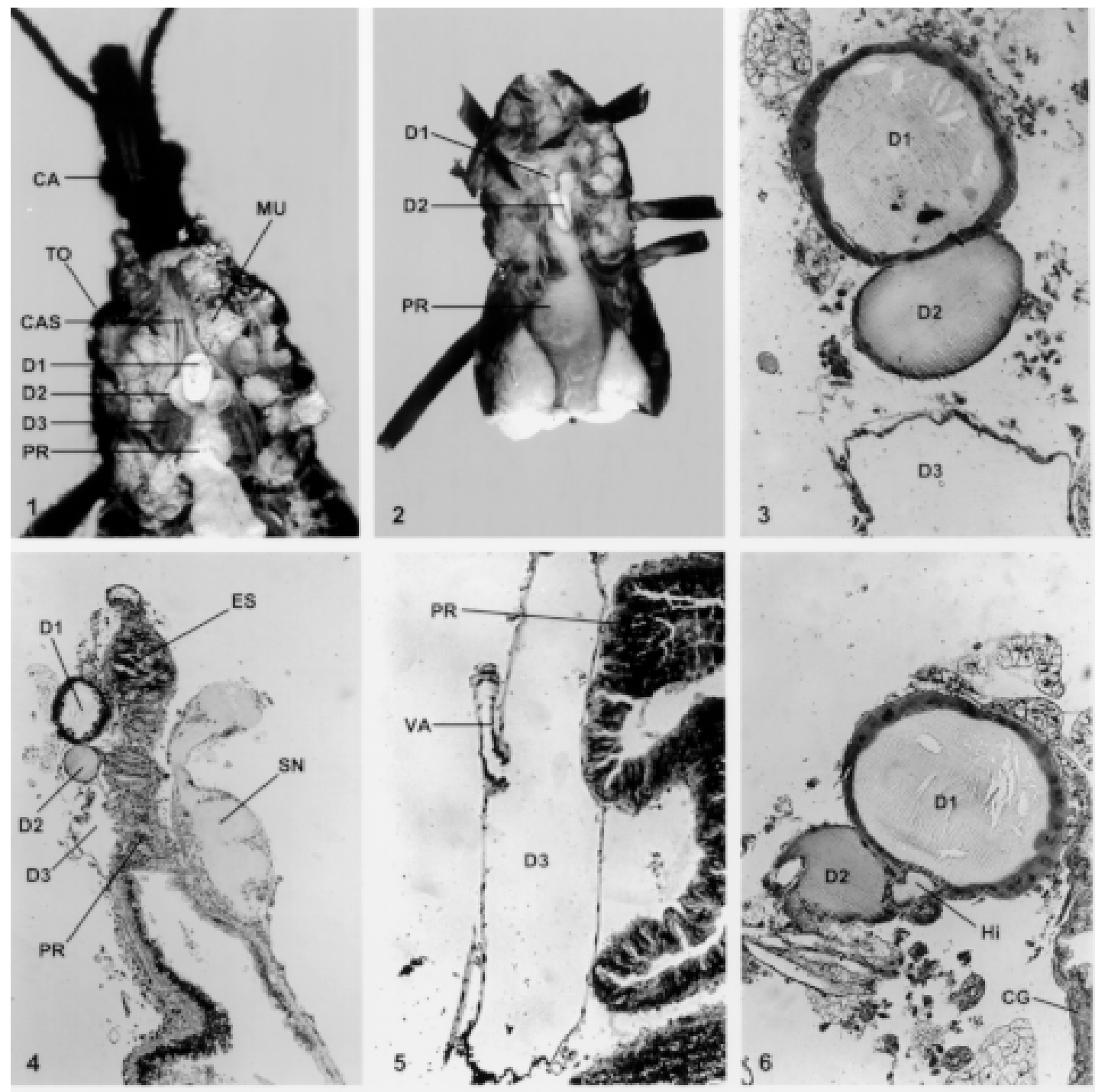

Fig.1: vista interna das glândulas salivares em Triatoma vitticeps. CA: cabeça; CAS: canal salivar; D1, D2 e D3: glândulas salivares; MU: musculatura; PR: promesêntero; TO: tórax. Fig. 2: corte longitudinal pelo tórax de Rhodnius prolixus. D1 e D2: glândulas alongadas; PR: promesêntero. Fig. 3: corte transversal pelas glândulas salivares em T. vitticeps. Coloração: galocianina + cromotrop 2R; D1, D2 e D3: glândulas salivares. 250x. Fig. 4: disposição das glândulas salivares em relação ao aparelho digestivo e ao sistema nervoso. Coloração: hematoxilina + cromotrop 2R; D1, D2 e D3: glândulas salivares; ES: esôfago; PR: promesêntero; SN: sistema nervoso. 100x. Fig. 5: corte longitudinal pela glândula D3 em Panstrongylus megistus. Coloração: hematoxilina Delafield + eosina; D3: glândula; PR: promesêntero; VA: válvula. 250x. Fig. 6: corte transversal pelas glândulas D1, D2 e hilo em T. pessoai. Coloração: hematoxilina mallory; D1 e D2: glândulas salivares; CG: corpo gorduroso; H: hilo. 250x. 
que não ocorre nos pares D2 e D3. Toda a secreção dos pares D1, D2 e D3 é encaminhada para o hilo. A Fig. 11 mostra a disposição da D1, D2 e D3 em relação ao hilo e o promesêntero, após um corte histológico longitudinal de $5 \mu \mathrm{m}$ em $T$. vitticeps, onde notamos que os pares D1 e D2 não possuem vávulas, que controlam a saída da secreção para o hilo, como ocorre nas glândulas D3. A secreção da D1 e D2 passa através de diminutos poros do epitélio para o hilo (Fig. 12).
As glândulas D2 são mais curtas, arredondadas ou ovais e sempre próximas à reentrância da D1, como podemos observar na Fig. 12. O epitelio é baixo e bem definido. A secreção é do tipo apócrina, isto é, feita através de contínuo fluxo de "gotículas" de secreção saídas do citoplasma para a luz da glândula. Após é encaminhada para a câmara II do hilo (veja abaixo estrutura morfológica do hilo). É muito comum encontrarmos as glândulas D1 e D2 repletas de secreção e a D3 sempre isenta de secreção.

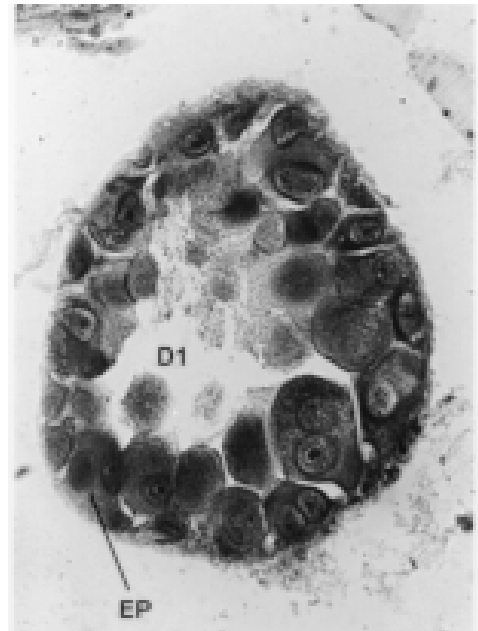

7

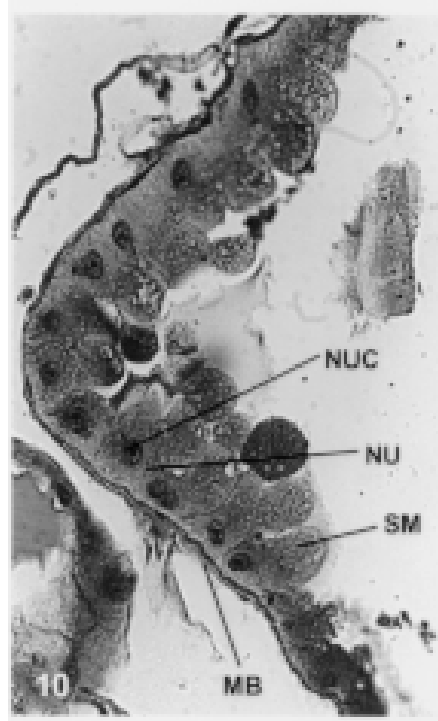

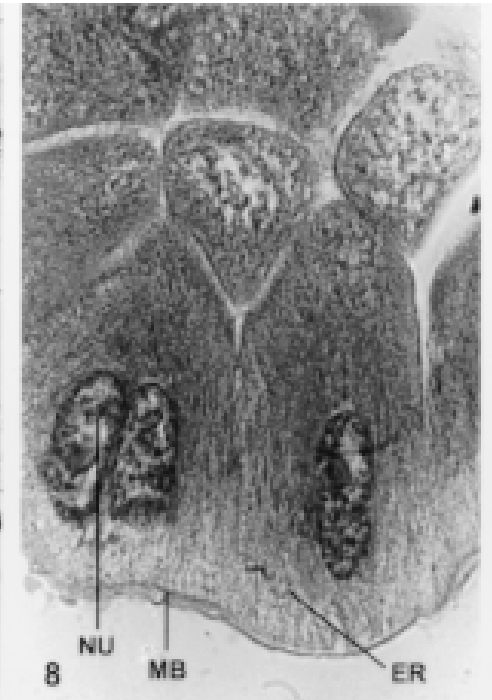
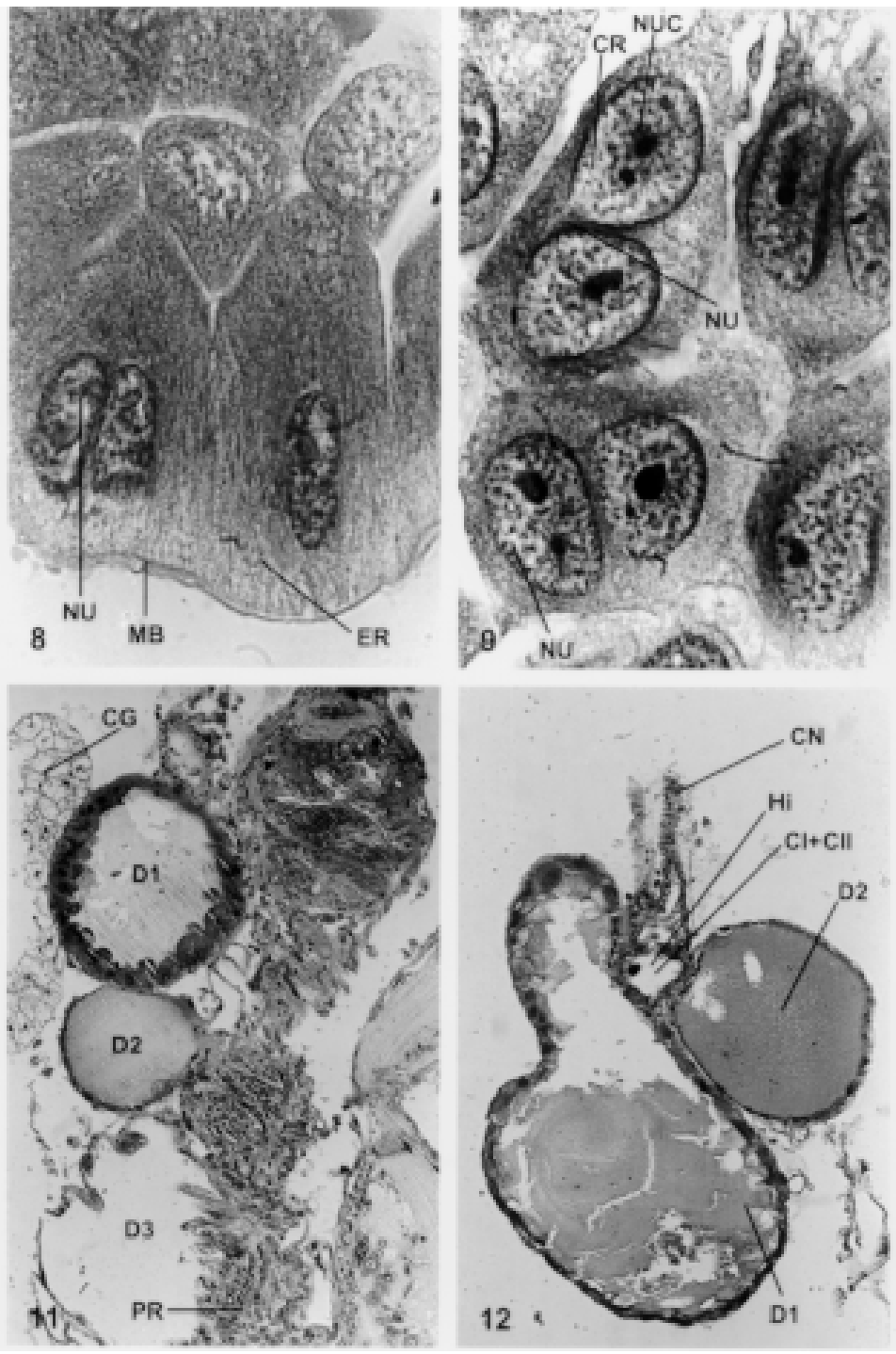

Fig. 7: corte histológico transversal pela glândula D1 em Panstrongilus megistus. Coloração: H.E.H.; EP: epitélio. 400x. Fig. 8: aspecto do epitélio da glândula D1 em Triatoma vitticeps. Coloração: H..E.H, ER: ergastoplasma; MB: membrana basal; NU:núcleo. 1000x. Fig. 9: células binucleadas das glândulas D1 em T. vitticeps. Coloração: H.E.H; CR: cromatina; NUC: núcléolos. 1000x. Fig. 10: corte longitudinal pela glândula salivar D1 em T. pessoai. Coloração: galocianina + cromotrop 2R; MB: membrana basal; NU: núcleo; NUC: nucléolos; SM: secreção merócrina. 100x. Fig. 11: corte longitudinal pelas glândulas salivares em T. pessoai. Coloração: galocianina + vermelho congo + orange GG; D1, D2 e D3: glândulas salivares; PR: promesêntero; CG: corpo gorduroso. 100x. Fig. 12: disposição das glândulas em relação ao hilo em T. pessoai. Coloração hematoxilina + verde naftol; CI: e CII: câmaras I e II; CN: canal secundário; HI: hilo. 100x. 
O par de glâdulas D3 é sempre maior, hialino, transparente, e afastado das anteriores (D1 e D2). O epitélio é baixo e sinuoso, com uma válvula característica que varia de posição nos gêneros Triatoma, Panstrongylus e Diptelogaster. A Fig. 13 dá uma vista geral da glândula D3 e as Figs. 14 e 15 mostram com nitidez a presença da válvula e o canal secundário que conduz a secreção destas glândulas para o hilo. Estas glândulas são muito sensíveis e ao contato com o líquido fixador logo se desfazem o que dificulta nossas observações. Porém, usando ácido ósmico segundo Romein (1928) e o bicloreto de mercúrio, conseguimos visualizar e analisar essas glândulas. A ausência de secreção no seu lúmen nos levou a acreditar que a liberação da secreção ali produzida seja imediata e nunca estocada, como vimos em D1 e D2. A vávula encontrada na D3 tem interessante estrutura, já mencionada por Barth (1954) durante seus estudos em T. infestans. Verificamos que entre os
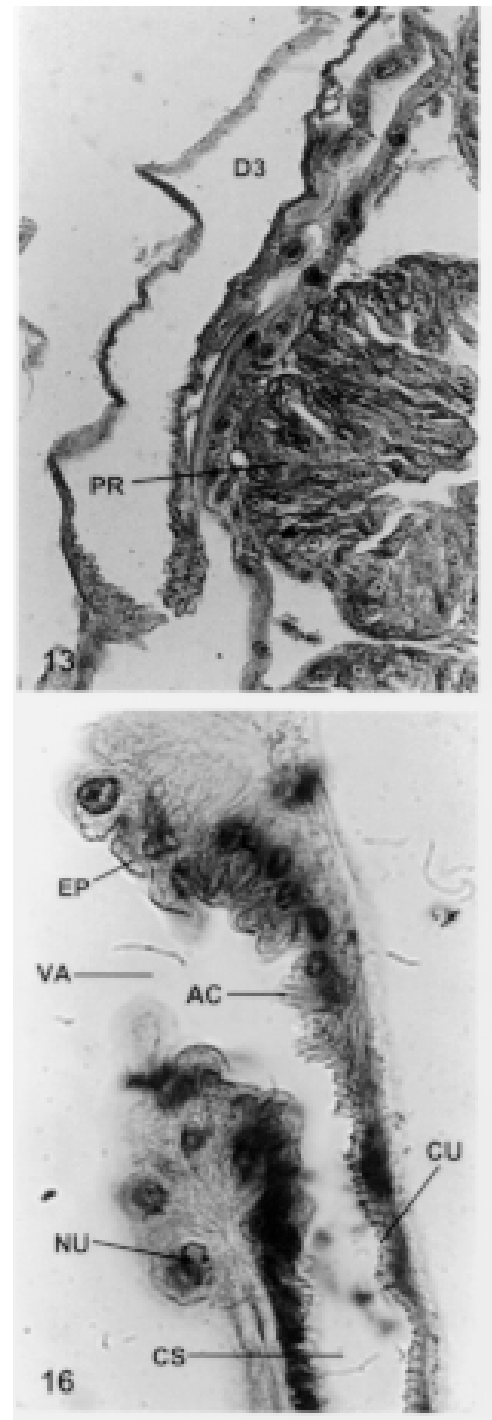
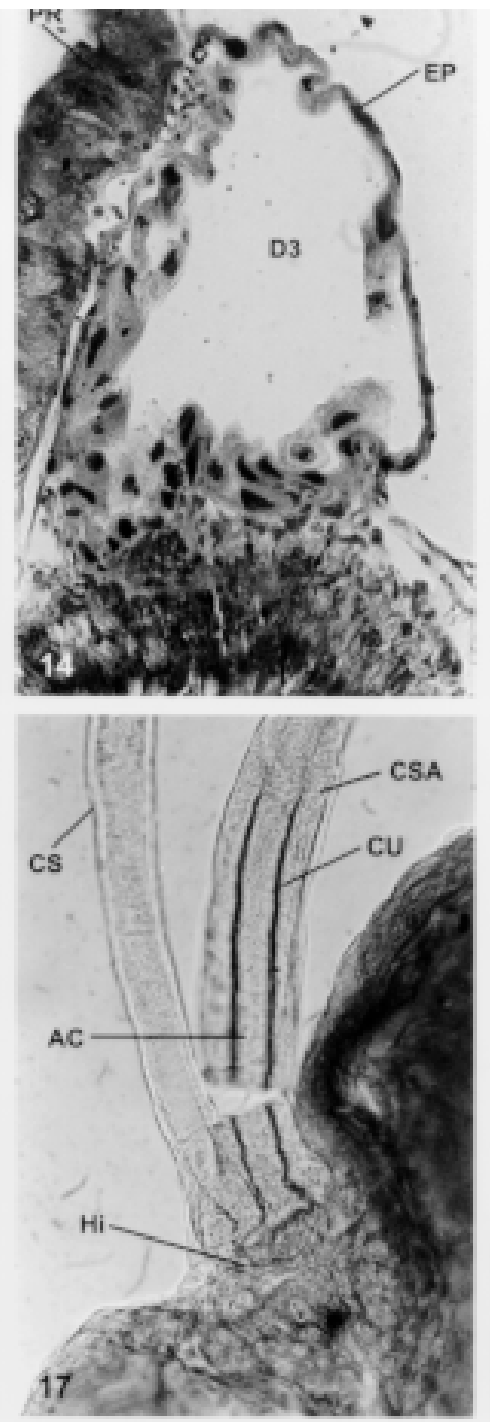
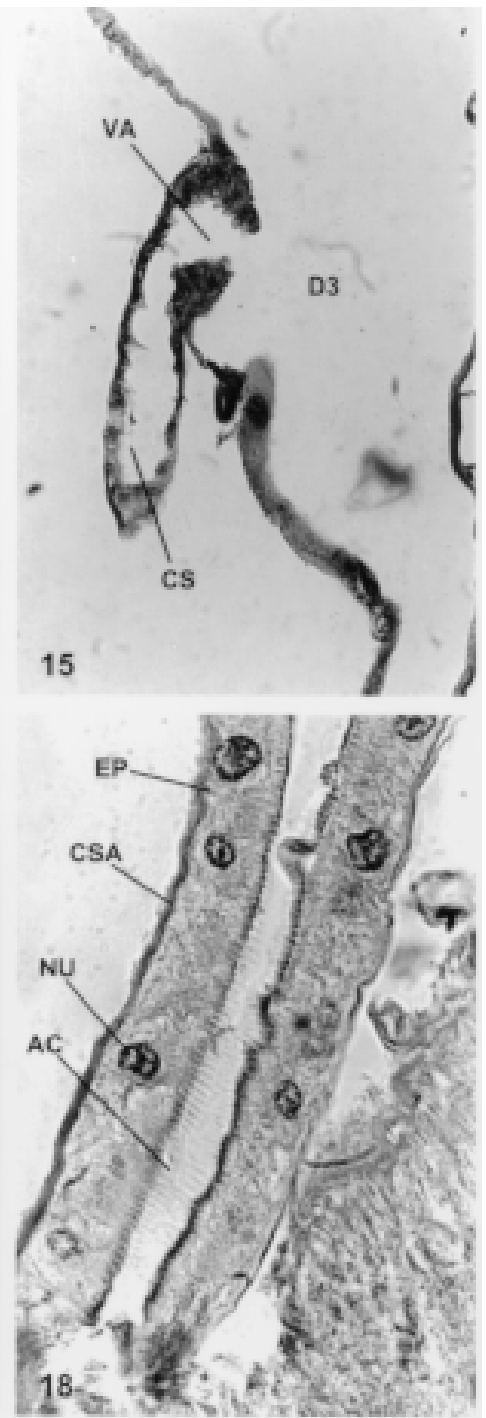

Fig. 13: corte longitudinal pela glândula D3 em Panstrongilus megistus. Coloração: H.E.H; D3: glândula salivar; PR: promesêntero. 100x. Fig. 14: aspecto histológico do epitélio da D3 em Triatoma vitticeps. Coloração: galocianina + eosina; D3: glândula salivar; EP: epitélio celular; PR: promesêntero. 250x. Fig. 15: vista geral da válvula da glândula D3 em P. megistus. Coloração: H.E.H; CS: canal secundário; VA: válvula. 250x. Fig. 16: corte longitudinal pela abertura da válvula D3 em P. megistus. Coloração: galocianina + cromotrop 2R; AC: anéis cuticulares; CU: cutícula; CS: canal secundário; EP: epitélio glandular; NU: núcleo; VA: válvula. 400x. Fig. 17: vista histológica dos canais em T. pessoai. Coloração: galocianina + vermelho congo + orange GG; AC: anéis cuticulares; CS: canal secundário; CSA: canal salivar; CU: cutícula; HI: hilo. 250x. Fig. 18: corte longitudinal do canal salivar em P. megistus. Coloração: H.E.H; AC: anéis cuticulares; CSA: canal salivar; EP: epitélio; NU: núcleo. 400x. 
triatomíneos estudados, esta glândula é mais nítida em P. megistus e T. vitticeps. O epitélio da D3 é sempre baixo, torna-se alto, espesso, com grandes núcleos na altura da vávula, como pode ser visto na Fig. 16, onde se mostra um corte histológico do início da válvula e do canal secundário. A localização desta válvula varia de posição. No gênero Triatoma está na parte lateral da glândula e em Pastrongylus na região distal. É relativamente difícil, através da simples dissecação em microscópio esterioscópio, determinar a presença da glândula D3 , daí alguns autores não as citarem em seus trabalhos. Devemos mencionar que este par está sempre presente nos gêneros Triatoma, Panstrongylus e Diptelogaster e ausente no gênero Rhodnius. A Fig. 16 ainda mostra o canal secundário com seus anéis circulares que impedem o fechamento do canal quando este se encontra em repouso. O canal secundário é muito parecido com o canal salivar (Fig. 17: CS e CSA), mas se diferencia do mesmo por ter menor diâmetro, cutícula fina, epitélio baixo e sinsicial. A trajetória feita por este canal secundário no interior do inseto é interessante. Tem início na glândula D3, segue em di-
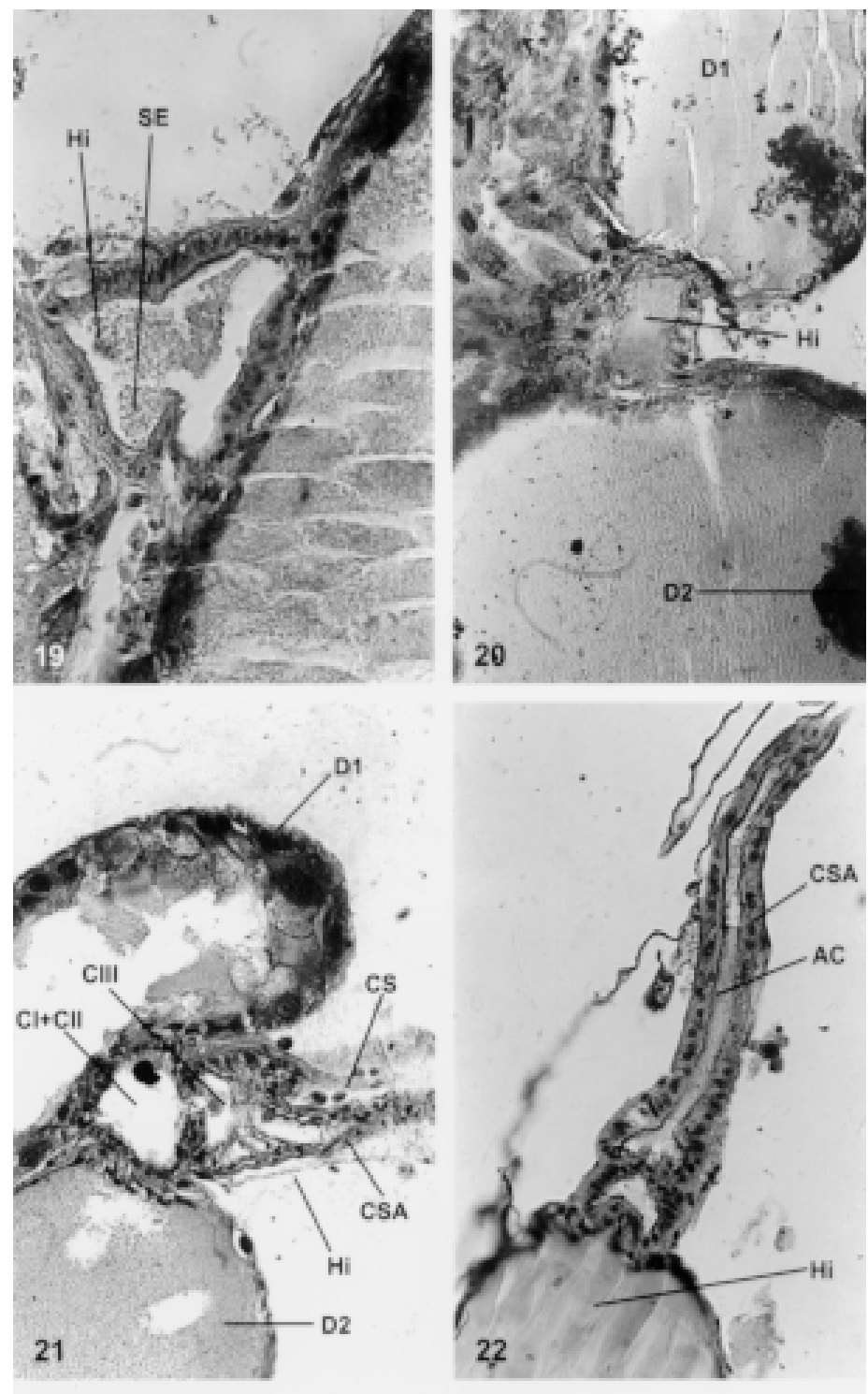

Fig. 19: aspecto do hilo em Triatoma, Panstrongylus e Diptelogaster. Coloração: H.E.H; HI: hilo; SE: secreção. 250x. Fig. 20: aspecto do hilo em Rhodnius. Coloração: galocianina + cromotrop 2R; D1 e D2: glândulas; HI: hilo. 250x. Fig. 21: estrutura morfológica do hilo em T. vitticeps. Coloração: H.E.H; CI, CII e CIII: câmaras I, II e III; CS: canal secundário; CSA: canal salivar.100x. Fig. 22: vista da glândula D3 próxima ao canal salivar em T. vitticeps. Coloração: H.E.H. + cromotrop 2R; AC: anéis cuticulares; CSA: canal salivar; HI: hilo. 100x. 
reção à região cefálica do inseto penetrando no início do foramem occipitalis e logo retorna ao tórax indo para o hilo, na câmara III, onde deposita a secreção. Este trajeto do canal secundário alonga o caminho da secreção para o hilo, desconhecendo-se a razão deste fato.

Em corte histológico longitudinal pelo canal salivar (Fig. 18: CSA) vemos o espesso epitélio salivar, núcleos ricos em cromatina e o citoplasma pouco denso que acompanha todo o canal. Os anéis cuticulares são bem evidentes, densos e numerosos. Sua disposição circular os diferenciam daqueles encontrados nas traquéias, que são espiralados e conhecidos como ctenídeos.

O hilo consiste de uma formação comum nos triatomíneos; sua forma é triangular nos gêneros Triatoma, Panstrongylus e Dptelogaster (Fig. 19) e arrendondada em Rhodnius (Fig. 20). Encontra-se sempre situado entre os pares de glândulas D1 e D2 funcionando como um reservatório das secreções. Na parte inferior do hilo vemos as câmaras CI e CII (Fig. 21) unidas em um só espaço e delimitadas pela válvula mediana do hilo que regula a passagem das secreções da D1 e D2 para a câmara superior CIII. Nesta região se une com a secreção da D3 e juntas seguem para o canal salivar (Fig. 22: CSA) em direção à bomba salivar e à hipofaringe. Devido a semelhanças histológicas do hilo com o canal salivar, alguns autores (Baptist 1941) consideram-no como um aumento de diâmetro da parte final do canal. Entretanto, a ausência de anéis cuticulares, o epitélio característico da parede do hilo e a formação da válvula mediana enfraquecem esta hipótese. Para melhores detalhes sobre a bomba salivar aconselhamos recorrer aos estudos de Barth (1954) em T. infestans e de Lacombe (1971) em Embolyntha batesi que citam a diversificação das glândulas salivares encontradas nos insetos.

\section{AGRADECIMENTOS}

A Professora Maria Celeste Dias Spata, do Departamento de Medicina Tropical, que separou e identificou os exemplares estudados, obtidos na criação original da saudosa Dra. Alina Szumlewicz e acompanhou o desenvolvimento do trabalho. Ao Laboratório Nacional e Internacional de Referência em Taxonomia de Triatomíneos, Insetário do Departamento de Entomologia, pelos triatomíneos recebidos. Ao Laboratório de Produção e Tratamento de Imagem do IOC, nas pessoas de Genilton Vieira, Heloísa Maria Diniz e Rodrigues Mexias. Ao Dr. Paulo Iide, ao Dr. Marcos Barbosa e ao biólogo Edalton Silva pelo incentivo que recebemos no decorrer do trabalho.

\section{REFERÊNCIAS}

Baptist BA 1941. The morphology and physiology of the salivary glands of Hemiptera-Heteroptera. Quart
J Microsc Sci 83: 91-139.

Barreto AH 1920. Notas entomológicas II. Estudo sobre a anatomia do gênero Triatoma. Aparelho Salivar. Mem Ins. Oswaldo Cruz 15: 127-130.

Barth R 1952. Estudos anatômicos e histológicos sobre a subfamilia Triatominae (Heteroptera-Reduvidae). 1a Parte: A cabeça do Triatoma infestans. Mem Inst Oswaldo Cruz 50: 70-196.

Bart R 1953. Métodos de trabalho na anatomia e histologia entomológica. Mem Inst Oswaldo Cruz 51: 95-189.

Barth R 1954. Estudos anatômicos e histológicos sobre a subfamilia Triatominae (Heteroptera-Reduvidae). IV Parte: O complexo das glândulas salivares de Triatoma infestans. Mem Inst Oswaldo Cruz 53: 517585.

Bocquet-Vedrine J 1970. Poliploide et multiplication amitotique des celules glândulaires cementaires chez crustcé cirripedia, operculé Balanus crenatus. CR Acad Sci 270: 506508.

Bordas L 1905. Sur ler glandes salivaires, cepháliques et metathoracique des qualquer hemipteres. CR Acad Sci CXL: 595-597.

Bugnion E, Popoff N 1908. Les apparielle salivaire des hemipterés. Arch 2001 D'anat. 10: 227-269.

Fauré-Fremiet 1910. Contribuition a l'etude des glands labiales des Hydrocorises. Ann Sci Natures (Zool) 9: 217-240.

Garcia ES, Mello CB, Ajambuja P, Ribeiro JM 1994. Rhodnus prolixus. Salivary antihemostatic components decrease with Trypanosoma rangeli infection. Exp Parasitol 78: 287-298.

Konig Jr B, Masuko TS, Rosemberg B 1993. Scannig electron microscopy of the Rhodnius neglectus labial salivary glands after starvation. Anat Anz 175: 411-416.

Lacombe D 1962. Estudos anatômicos e histológicos sobre a sub-família Triatominae. Parte XVIII. Sistema traqueal do Triatoma infestans. Mem Inst Oswaldo Cruz 60: 365-387.

Lacombe D 1971. Anatomy and histology of Embolyntha batesi Mac Lachlan 1877. Mem Inst Oswaldo Cruz 69: 331-396.

Pearse AG 1996. Histochemistry. Theorical and applied, J A Churchill Ltd, London, 998 pp.

Pereira MH, Souza ME, Vargas AP, Martins MS, Penido CM, Diotaiuti L 1996. Anticoagulant activity of Triatoma infestans and Panstrongylus megistus saliva (Hemiptera/ Triatominae). Acta Trop 61: 255-261.

Puri IM 1924. Studies on the anatomy of Cimex lectularius L. Parasitology 16: 84-97.

Ribeiro JC 1987. Role of salivar in blood feeding by arthropods. Ann Rev Entomol 32: 463-478.

Ribeiro JM, Schineider M, Isaías T, Jurberg J, Galvão C, Guimarães JA 1998. Role of salivary antihemostatic components in blood feeding by triatomine bugs (Heteroptera). J Med Entomol 35: 599-610.

Romein B 1928. Guia Formulário de Técnicas Histológicas, Ed. Barcelona, Madrid, 722 pp.

Santos CM, Jurberg J, Galvão C, Lent H 1997. Análise morfológica comparativa do complexo salivar dos Triatominae (Hemiptera: Reduviidae). Entomol Vect 
4: $155-162$.

Stark KR, James AA 1996. The salivary glands of diseases vectors, p. 333-348. In BJ Beaty e WC Marquardt (eds.), The Biology of Disease Vectors, Univ Press of Colorado, Niwot.

Sun J, Yuda M, Miura K, Chinzei Y 1998. Characterization and cDNA cloning of a hemoprotein in the salivary glands of the blood-sucking inset, Rhodnius prolixus. Insect Biochem Mol Biol 28: 191-200.

Thompson SW 1966. Selected Histochemical and Histological Methods, Charles C. Thomas, Illinois, XI+1639 pp.

Weber H 1930. Biologie der Hemipteren, J Springer, Berlin, 543 pp.

Wigglesworth VB 1939. The Principles of Insect Physiology, Met. Co. Ltd, London, VIII+434 pp., 316 figs. 